\title{
The association between physical activity and hypertension among HIV positive and negative populations in rural South Africa.
}

Benjamin Zebita Mayasi

University of freiburg

Gil Nelson

University of the Witwatersrand

Kerstin Klipstein-Grobusch

Universiteit Utrecht

Asaga Mac Peter ( $\sim$ pasaga123x@gmail.com )

Albert-Ludwigs-Universitat Freiburg https://orcid.org/0000-0003-0038-8896

Research article

Keywords: HIV/AIDS, Hypertension, South Africa, Physical Activity

Posted Date: January 10th, 2020

DOl: https://doi.org/10.21203/rs.2.20576/v1

License: (c) (i) This work is licensed under a Creative Commons Attribution 4.0 International License.

Read Full License 


\section{Abstract}

\section{Background}

Hypertension is a public health problem in sub-Saharan Africa, with considerable under- diagnosis, poor management, and lack of community-wide preventive strategies. Hypertension is a common condition in South Africa and is a risk factor for heart attacks, stroke, left ventricular hypertrophy, renal disease, and blindness. In this research study, we identify and examine the trend of physical activity within South Africa's HIV-positive and negative rural communities.

\section{Methods}

This was a secondary analysis of cross sectional survey data from the Agincourt Health and SocioDemography Surveillance System Site (AHDSS) that were collected over a period of 10 months, from August 2010 to May 2011. All 4436 individuals participating in the AHDSS survey were included. The inclusion criteria for the survey were from aged 15 years or older, and a permanent resident according to the 2009 census. The participants were interview (approximately 45 minutes) on chronic disease risk factor [applying] a questionnaire; anthropometric measurement were taken, blood pressure (BP); point-of care analysis of lipids and glucose; and dried blood spot collections for HIV and ELISA testing were conducted.

\section{Results}

The research findings showed a significant/strong association between hypertension and HIV in both univariate and multivariate analysis. In many developed countries, the prevalence of hypertension among HIV-positive individuals varies between $8 \%$ and $39 \%$. A study in Kenya reported a lower prevalence of $7.4 \%-11.2 \%$, compared with $22.1 \%-32.2 \%$ in study.

\section{Conclusion}

Hypertension is common in HIV-infected adults and is likely because of a combination of known risk factors, levels of physical activities/sedentary way of life, HIV-specific factors, and ART. There is a need for broad, global cohort studies to improve our knowledge and understanding of the above named subject matter, interventional studies are needed to discover new approaches in hypertension and HIVinfected individuals in South Africa and sub-Saharan Africa to avoid and manage hypertension and hypertension-related cardiovascular diseases among HIV infected persons especially in this era of epidemiologic and demographic transition in LMICs.

\section{Background}

Hypertension, also known as high or raised blood pressure, is a condition in which the blood vessels have persistently raised pressure. Hypertension is a public health problem in sub-Saharan Africa, with considerable under diagnosis, poor management, and lack of community-wide preventive strategies [1]. 
Hypertension is a common condition in South Africa and is a risk factor for heart attacks, stroke, left ventricular hypertrophy, renal disease, and blindness [2]. People who have hypertension are usually unaware that they have the condition, unless their blood pressure (BP) has been measured at a healthcare facility. It is therefore frequently referred to as a silent epidemic. Consequently, hypertension is universally inadequately treated, resulting in extensive target-organ damage and premature death(1). Hypertension frequently co-exists with other risk factors for chronic diseases of lifestyle (CDL), such as diabetes, obesity and physical inactivity. These interrelationships of hypertension with other CDL risk factors and the various possible targets organs that can be influenced by uncontrolled hypertension result in a diverse picture that has an impact on the South Africa population [2]. Physical inactivity in particular, has been related to increased risk of hypertension.

Over the past few decades, the physical activity patterns in the developing regions of the world have been changing from labour-intensive lifestyles to more sedentary and less physically demanding activities facilitating the development of CDL such as hypertension [3]. Physical activity has been shown to be an effective intervention to decrease BP the more one exercises, the greater the reduction in the risk of hypertension. This is because increase effective cardiovascular circulation of blood reducing possibility of blood vessel occulation. The paucity of data on physical activity and hypertension and of populationbased evidence on the association of physical activity with hypertension in the African continent hinders the implementation of effective preventive and control strategies [1].

The prevalence of hypertension among HIV negative people and among HIV positive people, ARV-naïve persons have been compared in several cross-sectional studies.

Therefore, studying the association between physical activity and hypertension is important, since these can help to inform policies and educate people and improve health service delivery and further research into drug and diagnostic development.

\section{Methods}

\section{Study Design}

This was a secondary analysis of cross-sectional survey data from the Agincourt Health and SocioDemography Surveillance System Site that were collected over a period of 10 months, from August 2010 to May 2011.

\section{Study setting}

The Agincourt Health and Socio-Demography Surveillance Site (AHDSS) monitors 90,000 people in 15,500 households living in 27 villages and is operated by the Medical Research Council (MRC)/Wits Rural Public Health and Health Transitions Research Unit. The site is situated in Northeast South Africa in the Bushbuckridge sub district of Ehlanzeni in Mpumalanga province. The site has annually recorded 
information on death, births, migration, marital status, education attainment, employment and socioeconomic in this population since 1992 [24].

\section{Study Population}

All individuals participating in the AHDSS survey that was conducted from August 2010 to May 2011. This included 1820 males and 2684 females (HIV positive and HIV negative): total of 4504 . The inclusion criteria for this survey were age 15 years or older, and a permanent resident according to the 2009 census round. The survey included an oversample of 280 adults over 50 from a prior study of older people, the 2006 study on Global and Adult Health (SAGE) that studied the health and well-being of a sample of 425 adults aged $50+[25]$.

\section{Method used in primary study}

The survey included 7662 individuals from a population of 34,413 using the 2009 HDSS census round sampling frame.

All sampled persons were visited by interviewers trained in the field research protocol, HIV counseling, and collection of dried blood spots (DBS) up to three times in their homes. The home interview (approximately 45 minutes) included a chronic disease risk factor questionnaire; anthropometric measurements including height, weight, hip circumference and blood pressure (BP); point-of care analysis of lipids and glucose and dried blood spot collections for HIV [25].

\section{Physical activity assessment}

Physical activity was assessed by questionnaire asking, in detail, frequency and duration of physical activity for work, transportation and leisure during a typical week (see appendix 1 ). Weekly physical activities were subsequently categorized into six groups: vigorous activities, moderate activities, travel to and from places, recreational activities, sedentary behavior and work.

\section{Blood pressure measurement}

Blood Pressure was measured three times on the right arm of the seated respondent using an automated recording instrument OMRON R6 Wrist Blood Pressure Monitor. The averages of the second and third systolic Blood Pressure (sBP) and diastolic Blood Pressure (dBP) measurements readings were used to estimate dBP and sBP Height was measured in centimeters $(\mathrm{cm})$, weight was measured in kilograms $(\mathrm{kg})$, waist circumference was measured in centimeters $(\mathrm{cm})$ and the hip circumference was measured in centimeters $(\mathrm{cm})$ [26]. And used to calculate body mass index (weight divided by squared height) and waist-to-hip ratio.

Blood pressure was classified following standards recommend by WHO, Normal Blood pressure was defined respectively for Systolic BP $120 \mathrm{mmHg}$ and Diastolic BP $80 \mathrm{mmHg}$. For Pre Hypertension was also defined for Systolic BP $>120 \mathrm{mmHg}$ and $<139 \mathrm{mmHg}$ and Diastolic BP $>80 \mathrm{mmHg}$ and $<89 \mathrm{mmHg}$. 
For Hypertension Systolic BP >140 mmHg and Diastolic BP $>90 \mathrm{mmHg}$ or use of antihypertensive medication [27].

\section{Ascertainment of HIV Status}

Informed consent was obtained prior to study enrolment. A confidential interview was conducted where participants were asked about contraceptive history for females and circumcision history for males. For HIV testing, blood spots were tested by using screening assay Vironostika Uniform 11 (Biomerieux, France), and positive results were confirmed by the SD Bio line HIV ELISA test as it is the standard diagnostics test for HIV. A third assay was done in cases where the screening and confirmatory tests did not match, and this was used to determine the final result of the test following WHO criteria [25].

\section{Assessment of Potential Confounders}

Anthropometric measurement assessed BMI, categories of WHR, were measured at all visits while the participant wore light clothing and no shoes. Elevated blood glucose, elevated lipids were also measured at each visit. Gender, age, union status, education, smoking status, alcohol intake and History of chronic diseases were assessed by using questionnaire during interview the participants that were asked about smoking status and alcohol intake.

\section{Data Analysis}

\section{Analysis variables}

\section{Exposure variables:}

Vigorous work activity, vigorous activities, moderate activities, travel to and from places and sedentary behavior.

\section{Outcome variables:}

Hypertension and HIV status

\section{Potential confounders' variables:}

Gender, age , union status, education, BMI, elevated blood glucose, categories of WHR, elevated lipids, smoking status, alcohol intake and History of chronic diseases

\section{Statistical Analysis}

\section{Data cleaning}

Data cleaning and all data analysis were done using STATA 13. Standard data cleaning procedures to identify values on variables were carried out by conducting checks on all extracted variables to determine 
the extent of missing values and duplicated variables. (A 20\% cut off point was used to delete observations with missing values and above $20 \%$ was retained).

\section{Generating new variables in the data and coding}

We generated three categories of smoking status and alcohol consumption: as never, past and current. History of chronic diseases were generated with two categories; Yes and No, every participant who had diabetes and who was diagnosed with stroke were included under the category yes. We generate a new variables called blood pressure that included systolic BP and diastolic BP, and were coded with three categories: low, normal and high. We generate a new variable called Hypertension with two categories: yes and no. categories of BMI (body mass index) Weight/height2 were coded into four categories: underweight, normal weight, overweight and obese, the participants with the BMl; less than 18.5 were considered as underweight, 18.5 - 24.9 were considered as normal weight, $25-29.9$ were consider as overweight and the BMI of 30+ were considered as Obese. Education was categorized into four categories: none, primary, secondary and tertiary. Union status was categorized into three categories: single, in a relationship, and unknown.

\section{Analysis}

Description of the levels of physical activity among HIV positive and HIV negative individuals: Descriptive frequency tabulation was conducted to know and understand the distribution of physical activity according to HIV status.

Prevalence of hypertension was described using proportion and percentages. A chi square test was used to check an association between the two categorical variables. For continuous variables, summary statistics were used; two sample t-tests were used to compare the means of two continuous variables (e.g $\mathrm{BMI}$ and hypertension) after the normality assumption was conducted using graphical and shapiro-wilk test.

Bivariate and multivariable logistic regression analysis were used to test the study hypotheses and the factors influencing the associations of PA and Hypertension to HIV negative and HIV positive. In the multivariate analysis we adjusted for the covariates gender, age, union status, education, BMI, Smoking status, alcohol consumption, HIV status, and history of chronic diseases, categories of WHR, elevated lipids, blood glucose and physical activities. All these covariates were included based on a p-value of < 0.20 in the bivariate analysis.

\section{Ethical Statement}

The primary study was approved by the University of the Witwatersrand Human Research Ethics Committee (HREC clearance certificate number M10458) and Mpumalanga Provincial Research and Ethics Committee. This secondary study was approved by the University of the Witwatersrand Human Research Ethics Committee (clearance certificate number M140917). Permission to use the data was obtained from The Agincourt Health and Socio-Demography Surveillance Site (AHDSS). 


\section{Results}

\section{Baseline characteristics of the study population}

\section{Table 1. Characteristics of participants at baseline with and without Hypertension}

Tables 1 showing the baseline characteristics of the study participants, those with hypertension, 775 (43.2\%) were males, and 1,018 (56.8\%) females and those without hypertension, were $932(35.9 \%)$ males and $1,666(64.1 \%)$ females respectively.

\section{Table 2. Socio-demographic Characteristic}

Table 2 showing socio-demographic characteristics /indices of the study population with a total of 4436 participants, of these 1,716 [38.7\%] were males and 2720 [61.3\%] were females, among the males, 775 [45.4\%] were hypertensive and 932 [54.6\%] had no hypertension and among the females, 1,018 [37.9\%] were with hypertension and 1,666 [62.1\%] had no hypertension. There was a statistical significant relationship between gender and hypertension ( $p$-value 0.0001 ). Among the different age groups, as the age increases, the number of people with hypertension also increase. Subjects who were 70 years and above had the highest percentage of the disease.

The result demonstrated that the association between age and hypertension were significant (P-value $0.0001) .3,419[77.1 \%]$ of the subjects were single and 402 [9.1\%] were married or in a relationship. 615 [13.9\%] did not communicate their marital status.

Those without unfold marital status had the highest hypertensive subjects [73.8\%], while single subjects had a lowest rate of $57.5 \%$. The relationship between hypertension and marital status was statistically significant (P-value 0.000).

The study population had 2,320 (53.7\%) secondary school subjects and 159 [3.7\%] college/University students' participants. A total number of 1063 had no formal/informal education. Those with hypertension were [55.6\%] and had no basic education. The relationship between hypertension and education was statistically significant (P-value 0.000 ). Body mass index [BMI] showed that $312[7.4 \%]$ of the total population were underweight and 2053[48.5\%] had normal weight, 1023[24.2\%] were overweight and $844(19.9 \%)$ were obese. The obese population motif had $53.3 \%$ hypertensives. The relationship between the two variables was statistical significant (P-value 0.000$)$.

The total number of participants who consumed/drank alcohol were 2,512 [56.6\%], 1,049 [23.7\%] did in the past, and $874(19.7 \%)$ subjects currently drink alcohol. Those who presently consumed alcohol had a prevalence of [48.0\%] patients with hypertension and those who never drank had the lowest preponderance of [38.2\%].The relationship between alcohol consumption and hypertension was also statistically significant $(P$-value $=0.000)$. Smoking status had 3,768 $(85.0 \%)$ of non-smokers, those who smoked in the past were 298 (6.7\%) those that currently were 369[8.3\%]. The hypothesized test of association between smoking and hypertension was statistically significant (P-value= 0.000$)$. History of 
chronic diseases revealed that 197 [4.4\%] had the diseases and 4,239 [95.6\%] had no history of chronic infection. $58.16 \%$ of those with history of chronic diseases had hypertension and $40.02 \%$ had no hypertension. The relationship between these two variables was found to be significant (with a $p$ value of 0.0001).

\section{Table 3. Description of physical activity pattern among HIV positive and HIV negative}

Table 3 shows that among the 4435 of the study participants, 3327 [75.0\%] were HIV negative and 1108 [25.0\%] were HIV positive. Subjects who engaged in physical practices were 2847 , and $2100[73.8 \%$ ] of these subjects were active participants and were also HIV-negative, 747 [26.2\%] were HIV-positive and inactive or less active participants. 1588. 1227[77.3\%] were HIV negative and 361[22.7\%] of the 1588 population were HIV positive. Moderate activity subjects were 3613 [25.7\%] and were also HIV positive while 822 [21.8\%] of the population were inactive subjects and were also HIV positive. Those who exercise by travelling from one location (Active) to the other were $3887[25.5 \%]$ and $2896[74.5 \%]$ of them were HIV positive, $584[21.4 \%]$ of the participants were inactive and $431[78.6 \%]$ were HIV positive. The total population of subjects who partake in vigorous sports activities were $730[19.7 \%]$ and $586[80.3 \%]$ of these were HIV negative. Entrant who were inactive in vigorous exercise were 3705[26.0\%] and 2741[ 74.0\%] were HIV negative, meaning that more people were inactive in vigorous sports and were HIV positive compared to those who were active

\section{Table 4 Prevalence of Hypertension among HIV positive and HIV negative population}

Table 4 shows that, out of 4436 of the total population 1793 had hypertension and 1434 were HIV negative while 359 were had the HIV virus (Positive). 2598 study participants had no hypertension. Among the HIV negative gender groups, male subjects constitute 1356 [79.0\%] and females constitute $1972[72.5 \%]$ of the total population. $633[46.9 \%]$ and $801[41.1 \%]$ of the male and female participants had hypertension. $360[21.0 \%]$ males and $748[27.5 \%]$ females were HIV positive compared to subjects without the virus. HIV positive males had the highest [60.2\%] number of hypertensive individuals compared to those without the virus (HIV negative). HIV positive females had a lower number [29.6\%] of hypertensive individuals in contrast to HIV negative subjects. With respect to age demographic indices among HIV negative age groups, 15-19 years had the highest population 483 [96.2\%] and 40-44 years had the lowest $194[64.0 \%]$. The number of hypertensive individuals were higher $296[63.1 \%]$ in the age group 70 years above and the youngest age group had the lowest rate of hypertension 116 [24.3\%]. A similar result was also observed among HIV positive individual, where hypertension rate was highest in age group 70 years and above compared to the younger age group 35-39 years. Among the HIV negative and positive age groups, hypertension prevalence rate were higher in age group 15-19 years in HIV positive subjects with a difference of $2 \%$, although the lowest rate of hypertension were observed in the age group 20-24 years. Among marital status socio-demography subjects, HIV negative singles had the highest population 2623 [76.3\%] and those with unknown status had 453 [73.7\%] (Lowest). Individual with unknown status had the highest prevalence rate of hypertension $273[60.7 \%]$ and those subjects who were single had the lowest prevalence rate of $1042[40.1 \%]$. 
Subjects who were HIV positive had a prevalence of $35.1 \%$ individuals with hypertension, these was closely followed by the unknown status group. Those participants who tested positive for HIV had the higher prevalence of hypertension compared to the population of negatively assessed subjects. HIV negative participants had a larger population compared to HIV positive individuals among the social demographic determinant educational group. The secondary school educational strata had the largest population of HIV negative [59.0\%] and positive [40.8\%] individuals. The prevalence of hypertension was highest among high (Secondary) school subjects.

HIV negative individuals that had attained tertiary education had the lowest rate [36\%] of hypertension; the same was true for the HIV positive tertiary educated population [15\%] group. Among the body mass index categories, HIV negative normal weight had the highest number of participants 1506 [73.4\%] in relation to those who were underweight 230[73.7\%]. The prevalence of hypertension was very higher [54.5\%] among obese subjects and lower in people who are underweight [35.5\%]. Subjects who tested positive to HIV had a lower prevalence of hypertension compared to those who tested negative. The prevalence of hypertension was [26.8\%] among underweight individuals and [47.8\%] among subjects who had normal weight. Among alcohol consumers, the preponderance of hypertension was higher in HIV negative than in HIV positive subjects who drink alcohol. HIV negatives population had a prevalence rate of [40.7\%] individuals who had hypertension but never drink alcohol this is in contrast to [50.7\%] the prevalence among those who currently drinking alcohol. The prevalence of [39.8\%] was observed among HIV positive individuals compared to [10.8\%] among HIV negative subjects. Those who were HIV positive and consumed alcohol in the past had a relatively lower hypertension prevalence [33.8\%] compared to [43.6\%] in HIV negative individuals.

Individuals who were HIV negative and positive and never smoke were 2871 and they constituted $41.9 \%$ prevalence of hypertensive subjects and $31.3 \%$ of the total population of HIV negative and positive individuals. Hypertension incidence among those subjects who smoke in the past was $65.1 \%$ among HIVnegative subjects and $48.9 \%$ among HIV-positive subjects. Subjects that had no chronic disease record and were HIV-negative were 3164[74.6\%] in relation with HIV-positive subjects 1075[25.4]. Hypertension preponderance was more [60.1\%] among chronic disease subjects population of HIV negatives in contrast to [48.5\%] in HIV positives individuals. It was also observed that those who had no history of chronic disease had a prevalence of [42.6\%] among HIV negative subjects and [32.4\%] prevalence among HIV positive individuals

In summary, the association between the outcome variable (Hypertension) and the explanatory variables namely ,gender, age group, marital status (HIV negative), education (HIV negative), BMI, alcohol, smoking status and history of chronic disease (HIV negative) all had a statistically significant $p$-value of less than 0.05 at $95 \%$ confidence interval. It showed that there was a relationship/association existing between these individual variables and hypertension. However, education (among HIV positive), history of chronic diseases (among HIV positive) indicated a marginal statistical significance of p-value 0.070 and 0.053 respectively, marital status (among HIV positive) had a p-value of 0.635 which indicated there was no relationship/association with hypertension. 
Table 5. Univariate and multivariate analysis of Physical Activities and Hypertension among HIV positive and HIV negative individuals

The univariate analysis in table 5 shows that the odds of having hypertension was 1.04 times per every unit increase in vigorous work activity, which is marginally associated at $[95 \% \mathrm{Cl} ; 0.89 ; 1.20]$ and is not statistically significant with $p$ value of 0.626 . Moderate activity shows that $37 \%$ probability of having hypertension for a unit increase is statistically significant with a p-value of 0.001 [95\% Cl; $1.14 ; 1.65]$, multivariate analysis shows that moderate activity was a confounder with an odd ratio change difference of $20 \%$, it indicates a $10 \%$ odds of having hypertension and was statistically not significant with a pvalue; $0.386 ;[95 \% \mathrm{Cl} ; 0.89 ; 1.36]$. Travelling to and from places showed a 1.08 times chances of having hypertension which was not statistically significant p-value 0.488 [95\% Cl; $0.87 ; 1.34]$, but vigorous sports showed a $72 \%$ odds of having hypertension for each unit increase with a statistical significant difference of p-value; $0.001[95 \% \mathrm{Cl} ; 1.41 ; 2.09]$.

The multivariate analysis showed an odds of $87 \%$ chances of having hypertension for a unit increase in vigorous sports, and the result was statistically significant p-value; $0.013[95 \% \mathrm{Cl} ; 1.13 ; 3.08]$. Metabolic physical activity was a protective factor with an odds of 0.63 times of contracting hypertension and a unit increase which was statistically significant, The multivariate metabolic result shows a protective factor of 0.44 times and was statistically significant p-value 0.015 [95\% Cl: $0.23 ; 0.85$ ]. Metabolic physical activity shows a protective factor against hypertension compared to other variables which showed increase chances of having hypertension.

\section{Table 6: Association between physical activities and hypertension among HIV positive and HIV negative individuals, taking into account confounding factors for hypertension}

To investigate the association between physical activities and hypertension among HIV positive and HIV negative individuals in this group of individuals, taking into account confounding factors for hypertension.

In table 6, possible confounding factors were included in the analysis of the physical activities showing protection against hypertension. Multivariate analysis shows that a unit increase moderate activity was a protective factor, with a $26 \%$ less chances of having hypertension, which was statistically significant with a p-value of 0.043 [95\% $\mathrm{Cl} ; 0.56 ; 0.99]$. Vigorous sports shows an odd of 0.58 times in having hypertension, however this was not statistically significant p-value of 0.151 [95\% Cl: $0.27 ; 1.22]$. Univariate analysis shows that the odds of having hypertension was $28 \%$ greater in males compared to females, this difference was however statistically significant with a p-value of 0.001 at $95 \%$ confidence interval. Multivariate evaluation showed higher chances of having hypertension with an odd ratio of $15 \%$ greater in males compared to females and this variable was statistically significant p-value $0.001[95 \% \mathrm{Cl}$ : $1.67 ; 2.77]$. The result shows that males have higher chances of becoming hypertensive in contrast to females. Univariate analysis also showed that a unit increase in age had a $3 \%$ chance of contracting hypertension and it was statistically significant p-value 0.001 , while in multivariate interpretation, there was a $2 \%$ chance of hypertension and it was also statistically significant with a p-value of 0.001 [95\% Cl: 
$1.02 ; 1.03]$. Single demographic variable compared to those in a relationship status, showed a $42 \%$ chances of having hypertension and it was statistically significant with a p-value 0.003 [95\% Cl: 1.13; 1.79], with respect to univariate interpretation and adjusting for confounding factors, there was a $21 \%$ odds of having hypertension but this was not statistically significant, p-value of 0.235 [ $95 \% \mathrm{Cl}$ : 0.88 ; 1.65]. Individuals with unknown marital status in the unadjusted analysis showed an odd ratio of 2.09 compared to singles with a statistical significant difference p-value of 0.001 [95\% Cl: 1.72; 2.54], but when adjusted, there was a $9 \%$ increase in the odds of having hypertension although the difference was not statistically significant p-value of $0.534[95 \% \mathrm{Cl}: 0.82 ; 1.42]$. In summary, the results shows that singles were more protective against hypertension in correlation to those in relationships, who possess higher chances of having hypertension. During univariate evaluation, education was a protective factor against hypertension. Participants who achieved at least primary form of education had a $48 \%$ less chances of having hypertension when compared to individuals who had no form of education. This demographic characteristic was statistically significant $p$-value of 0.001 [95\% $\mathrm{Cl}: 0.47 ; 0.71]$. Subjects who had secondary school level of education had a better protective factor of $64 \%$ less chances against hypertension compared to non-educated individuals with a statistically difference which was significance p-value 0.001 [95\% confidence interval].

Those who acquired tertiary or university form of education had $64 \%$ less chances of hypertension compared to the illiterate population and there was a statistical significant difference with p-value 0.001 . When adjusted for possible confounders, the various level of educational status were all protective factors, although less protective compared to the unadjusted, it was however not statistically significant at $95 \%$ confidence interval, meaning that it was likely due to attributed chances. The results shows that the non-educated subjects had higher chances of hypertension (none protective).

There were milder chances of having hypertension per unit increase in BMI. Unadjusted analysis showed $8 \%$ odds of having hypertension with a significant difference p-value of 0.001 [95\% $\mathrm{Cl} 1.07 ; 1.10]$. Adjusting for BMI thereafter with other variables showed a $9 \%$ increase in the probability of having hypertension with a p-value 0.001 [95\% Cl: $1.07 ; 1.11]$. Unadjusted alcohol consumption showed a relative increase in the odds of having hypertension, however for those who were drinking in the past, the odds of having hypertension were 1.12 times compared to those who never drink at all. This was not statistically significant p-value of 0.131 [95\% Cl: $0.96 ; 131]$. Currently drinking subjects had a 1.53 times of acquiring hypertension and it was statistically significant p-value of $0.001[95 \% \mathrm{Cl}: 1.29 ; 1.82]$ compared to the subjects who never drink. The odds of having hypertension in participants who currently drink alcohol were significantly higher compared to those who drink in the past. After adjusting for alcohol consumption, those who drink in the past showed no relationship with hypertension with an odd ratio of 1.00 and it was not statistically significant. The population of currently alcohol consumers showed a $59 \%$ increase in the odds of having hypertension compared to subjects who never drink alcohol. The result was statistically significant with a p value of 0.001 at $95 \%$ confidence interval. Current alcohol consumers had higher chances of having hypertension compared to others. Smoking status variable when unadjusted showed higher chances of having hypertension for those who smoke in the past and a relative protective factor was conferred against hypertension for those who currently smoke. The odds of 
having hypertension in those who smoke in the past was 2.59 times compared to individuals who never smoke at all, with a statistical significance difference, $\mathrm{p}$-value of 0.001 [at $95 \% \mathrm{Cl}: 1.95 ; 3.42$ ]. The population that currently smoke showed a $6 \%$ protection against hypertension compared to those who never smoke at all, the result was however not statistically significance with a p-value of 0.642 . After the adjustment of confounders, the odds of having hypertension decreased 1.28 times in individuals who smoke compared to those who never smoke and the result was not statistically significant wit a $p$-value of 0.223 [ $95 \% \mathrm{Cl}: 0.87 ; 1.86$ ]. The population that current smoke showed a greater protection against hypertension. However, after adjustment an odd of $35 \%$ less chances of having hypertension was achieved with a statistical significant difference, with a p value of 0.014 [at $95 \%$ confidence interval].

These results therefore, showed that past smokers were at a very high chance/risk of hypertension in relation to current smokers as compared to those who never smoke at all during their life time. In comparing the waist size, those with increase waist size had the odds of $99 \%$ in contracting hypertension compared to the population with normal waist. The difference was statistically significant at $p$ value 0.001 with $95 \%$ confidence interval. The adjusted variables showed that there were only $40 \%$ chances (odds) of hypertension and it was statically significant [p-value of 0.004 and a $95 \%$ confidence interval of $1.12 ; 1.75]$. Those with abnormal or increased waist size within the population had higher chances of hypertension compared to normal waist size individuals. Individual members of the population with history of chronic diseases had odds of 2.84 times of contracting hypertension compared to those with no history of chronic diseases There was a statistical significant difference between the two variables [pvalue of 0.001 and $95 \%$ confidence interval $2.00 ; 4.0]$ ). After adjusting for possible confounders, the result shows that the odds of having hypertension in subjects with history of chronic diseases were 1.60 times compared to those without history of chronic diseases. But there was no statistical significant difference between the variables [p-value of $0.08 ; 95 \% \mathrm{Cl}: 0.94 ; 2.72$ ]. Unadjusted HDL cholesterol level shows increased odds of having hypertension with increased level of cholesterol. There was an odds of $30 \%$ chances of having hypertension when comparing a normal to low cholesterol levels. The result shows a statistical significant difference between the two [p-value of $0.02495 \% \mathrm{Cl}: 1.03$; 1.63], in high cholesterol levels, the odds were $57 \%$ greater chances of having hypertension compared to a normal cholesterol level. There was a statistical significant difference between high level cholesterol and low level [p-value of 0.00 at $95 \%$ confidence interval].

Multivariate analysis showed protection against hypertension in individual with normal cholesterol with a $17 \%$ less chances of hypertension and high cholesterol a $18 \%$ less chances of hypertension when compared to low cholesterol level individuals. The protection was not statistically significant [p-values of 0.250 and 0.226 respectively]. LDL lipid level showed no protection against hypertension. Unadjusted normal cholesterol subjects showed an odd of 1.43 times of suffering hypertension compared to subjects with low level cholesterol. There was a statistical significant difference [p-value of $0.005 ; 95 \% \mathrm{Cl}: 1.11$; 1.84]. High cholesterol level participants within the study population had a higher probability of suffering hypertension with $88 \%$ greater chances when compared to low cholesterol individuals with a significant difference of $95 \%$ confidence interval. The assessment of association between blood glucose and hypertension, the unadjusted variables showed odds of $8 \%$ greater chances of having hypertension with a 
statistically significant difference $[\mathrm{P} ; 0.001 ; 95 \% \mathrm{Cl}: 1.05 ; 1.11]$ for a unit increase in blood glucose level. After adjustment, blood glucose showed a relatively protective factor with a $2 \%$ less chances of hypertension; however it was not statistically significant.

P trend was adopted from metabolic physical activity, $\mathrm{p}$-value as a combined value to understand statistical significances relationship if any, for all the physical activity types with hypertension. A statistically significance relationship was attained in univariate analysis with a p-value of 0.001 meaning, there was an association between physical activity types and hypertension. After adjusting for possible confounders, a non-statistical significance p-value of 0.137 was achieved, indicating there was no association found between physical activity and hypertension.

\section{Discussion}

This study employed a cross sectional survey data from the Agincourt Health and Socio-Demography Surveillance System, it looked at the association between physical activity and hypertension among HIV positive subjects compared to HIV individuals in rural South Africa setting.

\section{Physical Activity association with Hypertension}

The results in the study showed that physical activity is associated with hypertension in rural South Africa communities and this is in consonance with similar different studies that were conducted in other parts of the world including other Africa nations [2].

There are some experimental evidence from interventional studies that further support this assertion/relationship between physical activity and hypertension.

It is an established fact that effects of exercise on blood pressure helps in preventing hypertension. It is also a well-documented and characterized element by various studies in recent years [2, 4]. Some other studies have shown that physical inactivity is a risk factor for hypertension, this is also an accepted view by many other researchers who worked across the globe, numerous consensus statements and recommendations have also been published $[3,4,5]$.

Most of the studies on hypertension risk and physical activity relied on questionnaire data to stratify participants by activity patterns, example, as sedentary, active or inactive. This categorization allows for comparison between the extremes of activity levels, results of such surveys showed higher rates of elevated BP in sedentary versus active individuals $[7,8,9,10,11,12]$. A few surveys however, showed that there is no difference in hypertension risk factors and levels of activity $[13,14,15]$.

\section{Hypertension in rural South Africa /sub-Saharan}

Hypertension have been shown to be associated with physical inactivity [6]. In population-based surveys, high rates of hypertension were found among older adults in rural and urban South Africa communities, $44.0 \%-52.0 \%$ among men and $51.6-60.4 \%$ among women in 1998 [7]. 
These assertion agrees with our research study/outcome. Older adults are more affected by hypertension, which is an established risk factor for cardiovascular disease [8].

Our results in the present study further support the above statement and other previous study findings on hypertension in rural South Africa and sub-Saharan Africa.

Our research study also revealed that women [56.8\%] had higher rate of hypertension in contrast to men [43.2\%] the reason [in the present study] could be that men are more physically active than women, the study also revealed that the risk of hypertension was more in older adults compared to younger ones, especially those between the age group 40 - 70 years above. The reason for such an anomaly could be that young adults are more physically active than older adults, and as a result offers a protective factor to younger adults to have nether risk of contracting hypertension in comparison to older adult subjects.

In the present study it was observed that education plays a critical role in suffering hypertension. Individuals with primary or no education had higher risks of developing hypertension compared to educated subjects (those who had acquired secondary and tertiary levels of education). Similar findings were elucidated by a study conducted by Martins Mpe [23]. BMI in the current study was found to play a major role among South African population who developed hypertension. It was observed that people with high BMI 25 -29.9 -30 and above (overweight and Obese) had higher rates of hypertension compare to those with normal BMI <24.9. This is in contrast to the findings of George et a I[24] where subpopulation groups among Chinese population was assessed for $\mathrm{BMI}$ and hypertension, where it was revealed that increased BMI had a positive correlation with hypertension. This could be attributed to environmental factors and life style as well as ethnicity and other socio-demographic indices.

Alcohol consumption and smoking socio-demographic factors impact and give rise to hypertension as revealed in our study. It was perceived that people with previous and current history of smoking or drinking showed a high rate of hypertension compare to those who never smoke. This result is in consonance with other previous studies conducted in South Africa and other part of the world [24].

The current study demonstrated that participants with chronic diseases have very high rates of hypertension compare to those without chronic diseases. This result is in agreement with other various documented research literature across Africa and other parts of Asia [24]. This could be attributed to many social determinants among the study population.

\section{Hypertension and HIV Status in South Africa/Sub-Saharan}

The research findings showed a significant/strong association between hypertension and HIV in both univariate and multivariate analysis, our study further revealed a total dissonance with other similar studies carried out in sub-Saharan countries where some results showed lack of association between hypertension and HIV. In many developed countries, the prevalence of hypertension among HIV-positive individuals varies between $8 \%$ and $39 \%$. $[19,20]$. This is similar to our findings. A study in Kenya reported a lower prevalence of $7.4 \%-11.2 \%$, [21] compared with $22.1 \%-32.2 \%$ in our study. These could be 
ascribed to vigorous nature and athletic exuberance of the East Africans in carrying out physical activities/exercises which results in lower prevalence of hypertension in Kenya [22].

\section{Limitation of the study}

The limitations of this research study is associated with the design of the primary project, the measurement of errors and misclassifications of physical activity. Furthermore, the list of all the confounders' in correlation to hypertension were not completely listed. Our study, like several other observational, epidemiologic studies, cannot prove causation, but it lays a foundation for projections and for testing the outcome of interventions in countries in the middle of epidemiologic/demographic transition.

\section{Conclusion}

Hypertension is common in HIV-infected adults and is likely because of a combination of known risk factors, levels of physical activities/sedentary way of life, HIV-specific factors, and ART. This study described the current evidence of physical activity associated with possible mechanisms of hypertension in HIV-infected and HIV negative individual population in South Africa in relation to physical activity and sedentary life style as well as other socio-demographic and other novel pathophysiologic mechanisms for hypertension in HIV-infected and non-infected adults population. Other factors include among others but were not treated in this study may include microbial translocation, chronic inflammation, immune suppression and reconstitution, viral tropism, lipodystrophy, adipokines, and HIV-related chronic renal diseases. There is a need for broad, global cohort studies to improve our knowledge and understanding of the above named subject matter. Therefore, interventional studies are needed to discover new approaches in hypertension and HIV-infected individuals in South Africa and sub-Saharan Africa to avoid and manage hypertension and hypertension-related cardiovascular diseases among HIV infected persons especially in this era of epidemiologic and demographic transition in LMICs.

\section{Abbreviations}

1. ACEl: Angiotensin-Converting Enzyme Inhibitor

2. AHDSS: Agincourt Health and socio-demography Surveillance Site

3. AIDS: Acquired Immunodeficiency Syndrome

4. ARB: Angiotensin Receptor Blocker

5. ARV: Anti-Retroviral

6. BB: Beta-Blocker

7. BMI: Body Mass Index

8. BP: Blood Pressure

9. CCB: Calcium Channel Blocker

10. CDL: Chronic Disease of Lifestyle 
11. cm: centimeters

12. dBP: diastolic Blood Pressure

13. DBS: Dried Blood Spots

14. FDC: Fixed Dose Combination

15. HAART: Highly Active Antiretroviral Therapy

16. HDSS: Health Demographic Surveillance Site

17. HIV: Human Immunovirus

18. HREC: Human Research Ethics Committee

19. Kg: kilo grams

20. MRC: medical research council

21. SAGE: study on global and adult health

22. Sbp: Systolic Blood Pressure

23. TDR: Special programme for research and Training in Tropical Diseases

24. WHO: World Health Organization

25. WHR: Waist Hip Ratio.

\section{Declarations}

\section{Ethical Approval and Consent to participate}

The research study was conducted in compliance with the ethical principles of the University of the Witwatersrand Human Research Ethics Committee (HREC clearance certificate number M10458) and Mpumalanga Provincial Research and Ethics Committee. This secondary study was approved by the University of the Witwatersrand Human Research Ethics Committee (clearance certificate number M140917). Permission to use the data was obtained from The Agincourt Health and Socio-Demography Surveillance Site (AHDSS). Written informed consent was obtained from all study participants. All participants were adult, those who were 15 years $-17^{\text {th }}$ years parental consent was obtained both written and verbal.

\section{Consent for publication}

Not applicable

\section{Availability of data and materials}

Data is not restricted, data are contained in the manuscript.

\section{Competing interests}

The authors declare that they have no competing interests 


\section{Funding}

This work was partly funded by WHO/TDR training grants 2014. They provided the funding fpr the research project to be undertaken

\section{Authors' contributions}

BMZ: Design the project, collected the data, and wrote the manuscript and contributed to the statistical analysis.

GN: Supervised/Contributed to statistical analysis and took part in the data collection and participated in all phases of the work, contributed to writing of the manuscript.

KKG [Kerstin Klipstein-Grobusch]: Supervised the data analysis and also contributed to writing of the manuscript.

PAM: Supervised the entire process/Manuscript, and also contributed to writing of the manuscript All authors read and approved the manuscript for submission.

\section{Acknowledgements}

We wish to express our immense gratitude to The Agincourt Health and Socio-Demography Surveillance Site (AHDSS and University of the Witwatersrand for their support.

\section{References}

1. NIH Consensus development panel on Physical Activity and Cardiovascular Health. JAMA 1996; 276 : 241-246.

2. American College of Sports Medicine. Physical activity, physical fitness, and hypertension. Med Sci Sports Exerc 1993; 25 i-x.

3. Pate RR et al. Physical activity and public health. JAMA 1995; 273: 402-407. | Article | PubMed | ISI | ChemPort |

4. Prentice RL. Dietary assessment and the reliability of nutritional epidemiology reports. Lancet 2003; 362: 182. | Article | PubMed | ISI |

5. Paffenbarger RS, Wing AL, Hyde RT \& Jung DL. Physical activity and incidence of hypertension in college alumni. Am J Epidemiol 1983; 117: 245-257. | PubMed |

6. Wei $\mathrm{M}$ et al. Low cardiorespiratory fitness and physical inactivity as predictors of mortality in men with type 2 diabetes. Ann Intern Med 2000; 132: 605-611. | PubMed| ChemPort |

7. Pereira MA et al. Physical activity and incident hypertension in black and white adults: the Atherosclerosis Risk in Communities Study. Prev Med 1999; 28: 304-312. | Article | PubMed | ChemPort | 
8. Bassett DR et al. Physical activity and ethnic differences in hypertension prevalence in the United States. Prev Med 2002; 34: 179-186. | Article| PubMed |

9. MacAuley D et al. Physical activity, physical fitness, blood pressure, and fibrinogen in the Northern Ireland health and activity survey. J Epidemiol Comm Health 1996; 50: 258-263. | ChemPort|

10. Nevill AM et al. Modelling the associations of BMI, physical activity and diet with arterial blood pressure: some results from the Allied Dunbar National Fitness Survey. Ann Hum Biol 1997; 24: 229-247. | PubMed| ChemPort |

11. Menotti $A$ et al. Cardiovascular risk factors as determinants of 25-year all-cause mortality in the seven countries study. Eur J Epidemiol 2001; 17: 337-346. | Article| PubMed | ChemPort |

12. Bauman A \& Owen N. Habitual physical activity and cardiovascular risk factors. Med J Australia 1991; 154: 22-28. | PubMed | ChemPort |

13. Montoye HJ, Metzner HL, Keller JB \& Epstein FH. Habitual physical activity and blood pressure. Med Sci Sports 1972; 4: 175-181. | PubMed | ChemPort |

14. Dawber TR et al. Environmental factors in hypertension. In: Stamler J, Stamler R, Pullman TN (eds). The Epidemiology of Hypertension Grune and Stratton: New York 1967; pp 255-287.

15. Kannel WB \& Sorlie PD. Some health benefits of physical activity. The Framingham Study. Arch Intern Med 1979; 139: 857-861. | Article | PubMed | ChemPort |

16. Garcia-Palmieri MR et al. Increased physical activity: a protective factor against heart attacks in Puerto Rico. Am J Cardiol 1982; 50: 749-755. | Article| PubMed | ChemPort

17. Jericó C, Knobel H, Montero M, et al. Hypertension in HIV-infected patients and related factors. Am J Hypertens. 2005;18:1396-1401.

18. Barbaro G. Metabolic and cardiovascular complications of highly active retroviral therapy for HIV infection. Curr HIV Res. 2006;4:79-85.

19. Bloomfield GS, Hogan JW, Keter A, et al. Hypertension and obesity as cardiovascular risk factors among HIV seropositive patients in Western Kenya. PLoS One. 2011;6:e22288.

20. van de Vijver SJ, Oti SO, Agyemang C, Gomez GB, Kyobutungi C. Prevalence, awareness, treatment and control of hypertension among slum dwellers in Nairobi, Kenya. J Hypertens. 2013;31:10181024.

21. Msemburi W, Pillay-van Wyk V, Dorrington RE, Neethling I, Nannan N, Groenewald P, Laubscher R, Joubert J, Matzopoulos R, Nicol E, Notilana B, Prinsloo M, Sithole N, Somdyala N and Bradshaw D. Second national burden of disease study for South Africa: Cause-of-death profile for South Africa, 1997 2010. Cape Town: South African Medical Research Council, 2014. ISBN: 978-1-920618-34-6.

22. Mortality and causes of death in South Africa, 2014: Findings from death notification / Statistics South Africa. Pretoria: Statistics South Africa, 2015

23. Martin T, Mpe. Cardiovascular disease in South Africa. South Africa hearth, Vol 7; 3. 2018

24. George C; Linderman, BS; Jiapeng, Lu; Xin Sun, MS; Wei Xu MS; Khurrum N; Asir MD; Wade Schutz; Lixin Jiang; Harlan M, Krumholz. Association of Body Mass Index With Blood Pressure Among 1.7 
Million Chinese Adults. JAMA Netw Open. 2018;1(4):e181271.

doi:10.1001/jamanetworkopen.2018.1271

\section{Tables}

Table 1. Characteristics of participants at baseline with and without Hypertension

\begin{tabular}{|l|c|c|c|c|}
\hline \multirow{2}{*}{} & \multicolumn{4}{|c|}{ Hypertension (N=4436) } \\
\cline { 2 - 5 } & \multicolumn{2}{|c|}{ Yes (N=1,793) } & \multicolumn{2}{c|}{ No (N= 2,598) } \\
\hline Variable & $\mathbf{N}$ & $\%$ & $\mathbf{N}$ & $\%$ \\
\hline Gender & & & & \\
Male & 775 & 43.2 & 932 & 35.9 \\
Female & 1,018 & 56.8 & 1,666 & 64.1 \\
\hline
\end{tabular}

Table 2. Socio-demographic Characteristic 


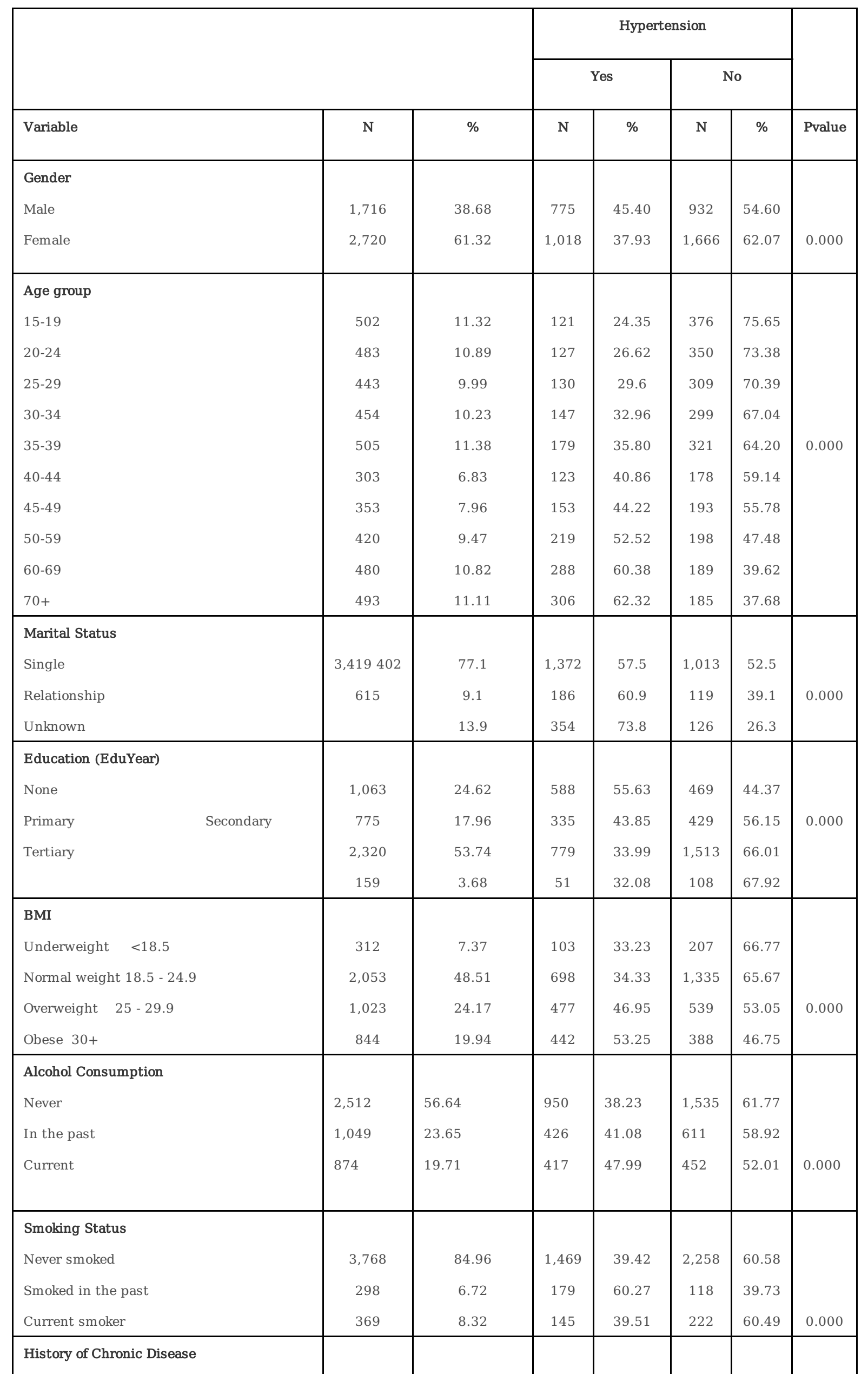

Page 20/26 


\begin{tabular}{|l|c|c|c|c|c|c|c|} 
Yes & 197 & 4.44 & 114 & 58.16 & 82 & 41.84 & \\
No & 4,239 & 95.56 & 1,679 & 40.02 & 2,516 & 59.98 & 0.000 \\
\hline
\end{tabular}

Table 3. Description of physical activity pattern among HIV positive and HIV negative

\begin{tabular}{|c|c|c|c|c|c|}
\hline \multirow[b]{2}{*}{ Physical activities } & \multicolumn{2}{|c|}{ HIV $-\quad(n=3,327)$} & \multicolumn{2}{|c|}{$\mathrm{HIV}+\quad(\mathrm{n}=1,108)$} & \multirow[b]{2}{*}{ Total (n) } \\
\hline & Frequency & Percentage & Frequency & Percentage & \\
\hline Vigorous work activity & & & & & \\
\hline Active & 2,100 & 73.8 & 747 & 26.2 & 2847 \\
\hline Inactive & 1,227 & 77.3 & 361 & 22.7 & 1588 \\
\hline Moderate activity & & & & & \\
\hline Active & 2,684 & 74.3 & 929 & 25.7 & 3613 \\
\hline Inactive & 643 & 78.2 & 179 & 21.8 & 822 \\
\hline Travel to /from places & & & & & \\
\hline Active & 2,896 & 74.5 & 991 & 25.5 & 3887 \\
\hline Inactive & 431 & 78.6 & 117 & 21.4 & 548 \\
\hline Vigorous sports & & & & & \\
\hline Active & 586 & 80.3 & 144 & 19.7 & 730 \\
\hline Inactive & 2,741 & 74.0 & 964 & 26.0 & 3705 \\
\hline MET quintile & & & & & \\
\hline 1 & 1,224 & 71.2 & 494 & 28.7 & \\
\hline 2 & 161 & 77.4 & 47 & 22.6 & \\
\hline 3 & 992 & 75.4 & 324 & 24.6 & \\
\hline 4 & 277 & 76.5 & 85 & 23.5 & \\
\hline 5 & 673 & 81.0 & 158 & 19.0 & \\
\hline
\end{tabular}

Table 4 Prevalence of Hypertension among HIV positive and HIV negative population 


\begin{tabular}{|c|c|c|c|c|c|c|c|}
\hline \multirow[b]{3}{*}{ Variable } & \multirow[b]{3}{*}{$\mathrm{N}(4,436)$} & \multirow[b]{3}{*}{$\%$} & \multicolumn{4}{|c|}{ Hypertension } & \multirow{3}{*}{$\begin{array}{c}\text { P- } \\
\text { value }\end{array}$} \\
\hline & & & \multicolumn{2}{|c|}{ Yes } & \multicolumn{2}{|c|}{ No } & \\
\hline & & & $\mathrm{N}(1,793)$ & $\%$ & $N(2598)$ & $\%$ & \\
\hline \multicolumn{8}{|l|}{ Gender (HIV) } \\
\hline \multicolumn{8}{|l|}{ Negative } \\
\hline Male & 356 & 79.0 & 633 & 46.9 & 717 & 53.1 & \\
\hline Female & 1,972 & 72.5 & 801 & & 1,149 & 58.9 & 0.001 \\
\hline \multicolumn{8}{|l|}{ Positive } \\
\hline Male & 360 & 21.0 & 142 & & 215 & 39.8 & \\
\hline \multirow[t]{2}{*}{ Female } & 748 & 27.5 & 217 & 60.2 & 517 & 70.4 & 0.001 \\
\hline & & & & 29.6 & & & \\
\hline \multicolumn{8}{|l|}{ Age group } \\
\hline \multicolumn{8}{|l|}{ Negative } \\
\hline $15-19$ & 483 & 96.2 & 116 & 24.3 & 362 & 75.7 & \\
\hline $20-24$ & 393 & 81.4 & 111 & 28.6 & 277 & 71.4 & \\
\hline $25-29$ & 296 & 66.8 & 95 & 32.4 & 198 & 67.6 & \\
\hline $30-34$ & 259 & 57.1 & 90 & 35.2 & 166 & 64.8 & \\
\hline $35-39$ & 281 & 55.6 & 102 & 36.7 & 176 & 63.3 & \\
\hline $40-44$ & 194 & 64.0 & 84 & 43.3 & 110 & 56.7 & \\
\hline $45-49$ & 237 & 67.1 & 108 & 46.4 & 125 & 53.6 & \\
\hline $50-59$ & 303 & 72.1 & 175 & 57.9 & 127 & 42.1 & \\
\hline $60-69$ & 411 & 85.6 & 257 & 62.8 & 152 & 37.2 & \\
\hline $70+$ & 471 & 95.5 & 296 & 63.1 & 173 & 36.9 & 0.000 \\
\hline \multicolumn{8}{|l|}{ Positive } \\
\hline $15-19$ & 19 & 3.8 & 5 & 26.3 & 14 & 73.7 & \\
\hline $20-24$ & 90 & 18.6 & 16 & 18.0 & 73 & 82.0 & \\
\hline $25-29$ & 147 & 33.2 & 35 & 24.0 & 111 & 76.0 & \\
\hline $30-34$ & 195 & 42.9 & 57 & 30.0 & 133 & 70.0 & \\
\hline $35-39$ & 224 & 44.4 & 77 & 34.7 & 145 & 65.3 & \\
\hline $40-44$ & 109 & 36.0 & 39 & 36.5 & 68 & 63.5 & \\
\hline $45-49$ & 116 & 32.9 & 45 & 39.8 & 68 & 60.2 & \\
\hline $50-59$ & 117 & 27.9 & 44 & 38.3 & 71 & 61.7 & \\
\hline $60-69$ & 69 & 14.4 & 31 & 45.6 & 37 & 54.4 & \\
\hline $70+$ & 22 & 4.5 & 10 & 45.5 & 12 & 54.5 & 0.002 \\
\hline \multicolumn{8}{|l|}{ Union Status } \\
\hline Single & 2,623 & 76.7 & 1,042 & 40.1 & 1,557 & 59.9 & \\
\hline Relationship & 252 & 62.7 & 119 & 47.4 & 132 & 52.6 & \\
\hline Unknown & 453 & 73.7 & 273 & 60.7 & 177 & 39.3 & 0.000 \\
\hline \multicolumn{8}{|l|}{ Positive } \\
\hline Single & 796 & 23.3 & 251 & 32.1 & 532 & 67.9 & \\
\hline
\end{tabular}




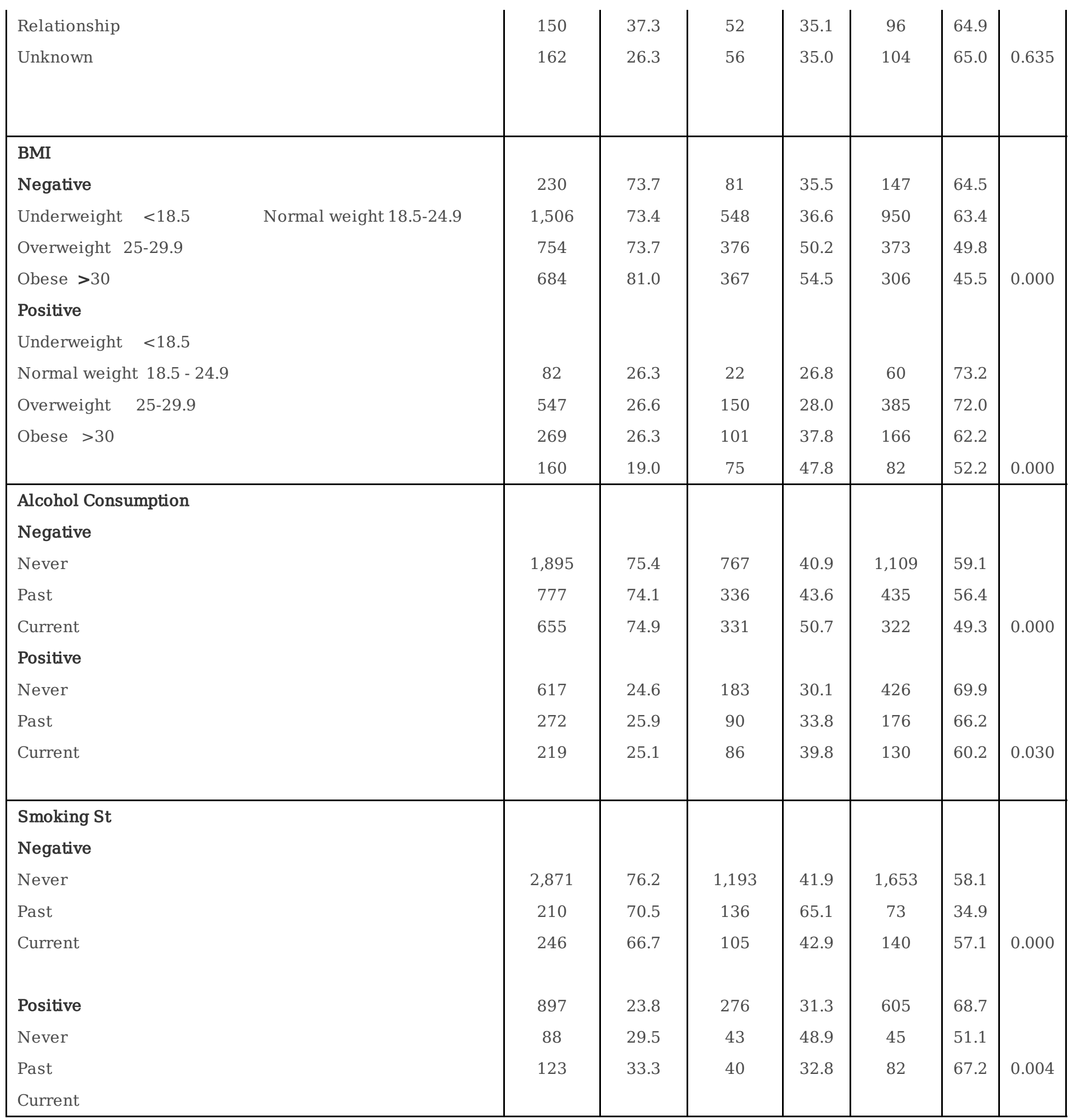

Table 5. Univariate and multivariate analysis of Physical Activities and Hypertension among HIV positive and HIV negative individuals 


\begin{tabular}{|c|c|c|c|c|c|c|}
\hline \multirow[t]{2}{*}{ Variables } & \multicolumn{3}{|c|}{ Univariable analysis of PA } & \multicolumn{3}{|c|}{ Multivariable analysis of PA } \\
\hline & Odds Ratio & $95 \% \mathrm{CI}$ & P-value & Odds Ratio & $95 \% \mathrm{CI}$ & P-value \\
\hline Vigorous work activity & 1.04 & $0.89 ; 1.20$ & 0.626 & & & \\
\hline Moderate activity & 1.37 & $1.14 ; 1.65$ & 0.001 & 1.10 & $0.89 ; 1.36$ & 0.368 \\
\hline Travel to from places & 1.08 & $0.87 ; 1.34$ & 0.468 & & & \\
\hline Vigorous sports & 1.72 & $1.41 ; 2.09$ & 0.001 & 1.87 & $1.13 ; 3.08$ & 0.013 \\
\hline Metabolic Physical Activity & 0.63 & $0.48 ; 0.82$ & 0.001 & 0.44 & $0.23 ; 0.85$ & 0.015 \\
\hline $\begin{array}{l}\text { Metabolic Quintile } \\
1\end{array}$ & Ref & & & Ref & & \\
\hline 2 & 0.97 & $0.69 ; 1.38$ & 0.881 & 1.07 & $0.77 ; 1.48$ & 0.680 \\
\hline 3 & 1.07 & $0.90 ; 1.28$ & 0.439 & 1.22 & $0.94 ; 1.57$ & 0.142 \\
\hline 4 & 1.22 & $0.93 ; 1.60$ & 0.153 & 1.68 & $1.10 ; 2.59$ & 0.017 \\
\hline 5 & 0.68 & $0.55 ; 0.83$ & 0.000 & 1.82 & $0.97 ; 3.43$ & 0.062 \\
\hline
\end{tabular}

Table 6: Association between physical activities and hypertension among HIV positive and HIV negative individuals, taking into account confounding factors for hypertension 


\begin{tabular}{|c|c|c|c|c|c|c|}
\hline \multicolumn{4}{|c|}{ Univariate analysis } & \multicolumn{3}{|c|}{ Multivariate analysis } \\
\hline Hypertension & Odds Ratio & $\mathrm{P}$ value & $(95 \% \mathrm{CI})$ & Odds Ratio & $P$ value & $(95 \% \mathrm{CI})$ \\
\hline Vigorous work activity & 1.04 & 0.626 & $0.89 ; 1.20$ & & & \\
\hline Moderate activity & 1.37 & 0.001 & $1.14 ; 1.65$ & 0.74 & 0.043 & $0.56 ; 0.99$ \\
\hline Travel to from places & 1.08 & 0.468 & $0.87 ; 1.34$ & & & \\
\hline Vigorous sports & 1.72 & 0.001 & $1.41 ; 2.09$ & 0.58 & 0.151 & $0.27 ; 1.22$ \\
\hline \multicolumn{7}{|l|}{ Metabolic quintile } \\
\hline 1 & Ref & & & Ref & & \\
\hline 2 & 0.97 & 0.881 & $0.69 ; 1.38$ & 0.94 & 0.794 & $0.61 ; 1.46$ \\
\hline 3 & 1.07 & 0.439 & $0.90 ; 1.28$ & 1.01 & 0.956 & $0.72 ; 1.41$ \\
\hline 4 & 1.22 & 0.153 & $0.93 ; 1.60$ & 1.14 & 0.638 & $0.65 ; 2.00$ \\
\hline 5 & 0.68 & 0.001 & $0.55 ; 0.83$ & 0.74 & 0.513 & $0.31 ; 1.81$ \\
\hline \multicolumn{7}{|l|}{ Gender } \\
\hline Female & Ref & & & Ref & & \\
\hline Male & 1.28 & 0.001 & $1.12 ; 1.46$ & 2.15 & 0.000 & $1.67 ; 2.77$ \\
\hline Age & 1.03 & 0.001 & $1.03 ; 1.04$ & 1.02 & 0.000 & $1.02 ; 1.03$ \\
\hline \multicolumn{7}{|l|}{ Marital Status } \\
\hline Single & Ref & & & Ref & & \\
\hline In relationship & 1.42 & 0.003 & $1.13 ; 1.79$ & 1.21 & 0.235 & $0.88 ; 1.65$ \\
\hline Unknown & 2.09 & 0.001 & $1.72 ; 2.54$ & 1.09 & 0.534 & $0.82 ; 1.47$ \\
\hline
\end{tabular}




\begin{tabular}{|c|c|c|c|c|c|c|}
\hline \multicolumn{7}{|l|}{ Education } \\
\hline None & Ref & & & Ref & & \\
\hline Primary & 0.58 & 0.001 & $0.47 ; 0.71$ & 0.81 & 0.153 & $0.61 ; 1.08$ \\
\hline Secondary & 0.34 & 0.001 & $0.29 ; 0.40$ & 0.79 & 0.114 & $0.60 ; 1.06$ \\
\hline Tertiary & 0.36 & 0.001 & $0.25 ; 0.53$ & 0.69 & 0.144 & $0.43 ; 1.13$ \\
\hline Bmi & 1.08 & 0.001 & $1.07 ; 1.10$ & 1.09 & 0.000 & $1.07 ; 1.11$ \\
\hline \multicolumn{7}{|l|}{ Alcohol Consumption } \\
\hline Never & Ref & & & & & \\
\hline Past & 1.12 & 0.131 & $0.96 ; 1.31$ & 1.00 & 0.943 & $0.79 ; 1.24$ \\
\hline Current & 1.53 & 0.001 & $1.29 ; 1.82$ & 1.59 & 0.001 & $1.21 ; 2.09$ \\
\hline \multicolumn{7}{|l|}{ Smoking Status } \\
\hline Never & $\operatorname{Ref}$ & & & Ref & & \\
\hline Past & 2.59 & 0.001 & $1.95 ; 3.42$ & 1.28 & 0.223 & $0.87 ; 1.86$ \\
\hline Current & 0.94 & 0.642 & $0.75 ; 1.19$ & 0.65 & 0.014 & $0.46 ; 0.92$ \\
\hline \multicolumn{7}{|l|}{ History chronic } \\
\hline No & s & & & Ref & & \\
\hline Yes & 2.84 & 0.001 & $2.00 ; 4.02$ & 1.60 & 0.081 & $0.94 ; 2.72$ \\
\hline \multicolumn{7}{|l|}{ Whr cat } \\
\hline Normal & Ref & & & Ref & & \\
\hline Elevate & 1.99 & 0.001 & $1.72 ; 2.30$ & 1.40 & 0.004 & $1.12 ; 1.75$ \\
\hline \multicolumn{7}{|l|}{ HDL Cholesterol } \\
\hline Low cholesterol & Ref & & & Ref & & \\
\hline normal cholesterol & 1.30 & 0.024 & $1.03 ; 1.63$ & 0.83 & 0.250 & $0.60 ; 1.14$ \\
\hline high cholesterol & 1.57 & 0.001 & $1.23 ; 1.95$ & 0.82 & 0.226 & $0.59 ; 1.13$ \\
\hline \multicolumn{7}{|l|}{ LDL Cholesterol } \\
\hline Low cholesterol & Ref & & & Ref & & \\
\hline normal cholesterol & 1.43 & 0.005 & $1.11 ; 1.84$ & 1.04 & 0.809 & $0.78 ; 1.38$ \\
\hline high cholestolerol & 1.88 & 0.001 & $1.58 ; 2.24$ & 1.19 & 0.102 & $0.97 ; 1.48$ \\
\hline Blood glucose & 1.08 & 0.001 & $1.05 ; 1.11$ & 0.98 & 0.398 & $0.94 ; 1.02$ \\
\hline *P trend & \multicolumn{3}{|c|}{0.001} & \multicolumn{3}{|c|}{0.137} \\
\hline
\end{tabular}

* adjusted for Gender, age, marital status,, educational attainment, body mass index, waist-to-hip ratio, smoking status, alcohol consumption, history of chronic disease, HDL Cholesterol, LDL Cholesterol, Blood glucose.

* P trend (Metabolic Physical activity P value for unadjusted and adjusted). 